\title{
Support for Online Learning at Rhodes University
}

\begin{tabular}{|c|c|c|}
\hline $\begin{array}{l}\text { Type of } \\
\text { support }\end{array}$ & Contact details & Links \\
\hline $\begin{array}{l}\text { IT Support: } \\
\text { Student } \\
\text { Helpdesk }\end{array}$ & $\begin{array}{l}\text { Email: } \\
\text { studentsupport@ru.ac.za }\end{array}$ & $\begin{array}{l}\text { https://www.ru.ac.za/student } \\
\text { networking/ }\end{array}$ \\
\hline RUconnected & Email: edtech@ru.ac.za & https://ruconnected.ru.ac.za/ \\
\hline $\begin{array}{l}\text { Academic } \\
\text { Support }\end{array}$ & $\begin{array}{l}\text { Please contact your lecturer/s } \\
\text { and/or tutor/s for any support } \\
\text { regarding specific course } \\
\text { content. }\end{array}$ & \\
\hline $\begin{array}{l}\text { Division of } \\
\text { Student Affairs }\end{array}$ & studentaffairs@ru.ac.za & $\begin{array}{l}\text { https://www.ru.ac.za/student } \\
\text { affairs/ }\end{array}$ \\
\hline $\begin{array}{l}\text { Counselling } \\
\text { Centre }\end{array}$ & $\begin{array}{l}\text { Email: } \\
\text { counsellingcentre@ru.ac.za }\end{array}$ & \\
\hline $\begin{array}{l}\text { International } \\
\text { Office }\end{array}$ & $\begin{array}{l}\text { Email: } \\
\text { internationaloffice@ru.ac.za }\end{array}$ & $\begin{array}{l}\text { https://www.ru.ac.za/internat } \\
\text { ionalisation/ }\end{array}$ \\
\hline $\begin{array}{l}\text { Registrar's } \\
\text { Division }\end{array}$ & $\begin{array}{l}\text { Melanie Appollis } \\
\text { Secretariat Assistant } \\
\text { registrar@ru.ac.za }\end{array}$ & $\frac{\mathrm{https}: / / \text { www.ru.ac.za/registra }}{\underline{\mathrm{r}} \underline{\mathrm{l}}}$ \\
\hline
\end{tabular}




\begin{tabular}{|c|c|c|}
\hline $\begin{array}{l}\text { Residential } \\
\text { Operations }\end{array}$ & $\begin{array}{l}\text { Heidi Smith } \\
\text { Residence Administrator } \\
\text { Email: } \underline{\text { h.smith@ru.ac.za }}\end{array}$ & \\
\hline Student Bureau & $\begin{array}{l}\text { Desiree Wicks } \\
\text { Manager Student Bureau } \\
\text { d.wicks@ru.ac.za }\end{array}$ & \\
\hline $\begin{array}{l}\text { Centre for } \\
\text { Postgraduate } \\
\text { Studies (CPGS) }\end{array}$ & $\begin{array}{l}\text { Siphokazi Mankayi } \\
\text { Assistant to the Director } \\
\text { s.mankayi@ru.ac.za }\end{array}$ & $\begin{array}{l}\text { https://www.ru.ac.za/postgra } \\
\text { duategateway/ }\end{array}$ \\
\hline Virtual Library & library@ru.ac.za & https://www.ru.ac.za/library/ \\
\hline $\begin{array}{l}\text { Support for } \\
\text { students with } \\
\text { disabilities }\end{array}$ & $\begin{array}{l}\text { Veronica Israel } \\
\text { v.israel@ru.ac.za }\end{array}$ & \\
\hline
\end{tabular}




\begin{tabular}{|c|l|}
\hline FACULTY OF HUMANITIES & Faculty Officer: \\
Karen Kouari \\
k.kouari@ru.ac.za \\
\hline FACULTY OF COMMERCE & $\begin{array}{l}\text { Commerce Faculty Officer: } \\
\text { Mrs Niki Searle } \\
\text { n.searle@ru.ac.za }\end{array}$ \\
\hline FACULTY OF SCIENCE & Faculty Administration Officer: \\
Ms Lusanda Klaas \\
\hline scisec@ru.ac.za \\
\hline FACULTY OF LAW & Secretary: \\
\hline FACULTY OF PHARMACY & Ms F Mwellie \\
& f.mwellie@ru.ac.za \\
\hline & Faculty Administrator: \\
\hline & I.emslie@ru.ac.za \\
\hline & S.asmal-motara@ru.ac.za \\
\hline
\end{tabular}




\begin{tabular}{|c|c|}
\hline Accounting & $\begin{array}{l}\text { Mrs Margie Hepple } \\
\text { m.hepple@ru.ac.za }\end{array}$ \\
\hline Afrikaans and Netherlandic Studies & $\begin{array}{l}\text { Mrs Hannelie Campher } \\
\text { h.rielly@ru.ac.za }\end{array}$ \\
\hline African Languages & $\begin{array}{l}\text { Mrs Hannelie Campher } \\
\text { h.rielly@ru.ac.za }\end{array}$ \\
\hline Anthropology & $\begin{array}{l}\text { Mrs Des Bekker } \\
\text { d.bekker@ru.ac.za }\end{array}$ \\
\hline Biochemistry, Microbiology & $\begin{array}{l}\text { Ms Lumka Mqingwana } \\
\text { I.mqingwana@ru.ac.za }\end{array}$ \\
\hline Biotechnology Innovation Centre & $\begin{array}{l}\text { Mrs Xolisa Freduah } \\
\text { x.matole@ru.ac.za }\end{array}$ \\
\hline Botany & $\begin{array}{l}\text { Ms Busi Goba } \\
\text { b.goba@ru.ac.za }\end{array}$ \\
\hline Business School & $\begin{array}{l}\text { Ms Aviwe Petsha } \\
\text { a.petsha@ru.ac.za }\end{array}$ \\
\hline $\begin{array}{l}\text { Centre for Higher Education Research, } \\
\text { Teaching and Learning }\end{array}$ & $\begin{array}{l}\text { Ms Nomfundo Siqwede } \\
\text { n.siqwede@ru.ac.za }\end{array}$ \\
\hline Chemistry & $\begin{array}{l}\text { Mrs Benita Tarr } \\
\text { b.tarr@ru.ac.za }\end{array}$ \\
\hline Chinese & $\begin{array}{l}\text { Mrs Hannelie Campher } \\
\text { h.rielly@ru.ac.za }\end{array}$ \\
\hline Classical Studies & $\begin{array}{l}\text { Mrs Hannelie Campher } \\
\text { h.rielly@ru.ac.za }\end{array}$ \\
\hline Computer Science & $\begin{array}{l}\text { Ms Michelle Coupe } \\
\text { m.coupe@ru.ac.za }\end{array}$ \\
\hline
\end{tabular}




\begin{tabular}{|c|c|}
\hline Drama & $\begin{array}{l}\text { Ms Vusiwe Mnyobe } \\
\text { v.mnyobe@ru.ac.za }\end{array}$ \\
\hline Economics and Economics History & $\begin{array}{l}\text { Mrs Chantelle van Eyssen } \\
\text { c.vaneyssen@ru.ac.za }\end{array}$ \\
\hline Education & $\begin{array}{l}\text { Ms Siyanda Bara } \\
\text { s.bara@ru.ac.za }\end{array}$ \\
\hline English Language and Linguistics & $\begin{array}{l}\text { Ms Vuyokazi Nkayi } \\
\text { v.nkayi@ru.ac.za }\end{array}$ \\
\hline Environmental Science & $\begin{array}{l}\text { Ms Nomvula Sitole } \\
\text { N.Sitole@ru.ac.za }\end{array}$ \\
\hline Extended Studies Unit & $\begin{array}{l}\text { Ms Denise Jeggels } \\
\text { d.jeggels@ru.ac.za }\end{array}$ \\
\hline Fine Art & $\begin{array}{l}\text { Ms Moira Japp } \\
\text { moira.japp@@ru.ac.za }\end{array}$ \\
\hline French & $\begin{array}{l}\text { Mrs Hannelie Campher } \\
\text { h.rielly@ru.ac.za }\end{array}$ \\
\hline Geography & $\begin{array}{l}\text { Mr Mthunzi Gumede } \\
\text { geography@ru.ac.za / } \\
\text { m.gumede@ru.ac.za }\end{array}$ \\
\hline Geology & $\begin{array}{l}\text { Mrs Ashley Goddard } \\
\text { a.goddard@ru.ac.za }\end{array}$ \\
\hline German & $\begin{array}{l}\text { Mrs Hannelie Campher } \\
\text { h.rielly@ru.ac.za }\end{array}$ \\
\hline History & $\begin{array}{l}\text { Mrs Xolisa Nontyi } \\
\text { x.nontyi@ru.ac.za } \\
\end{array}$ \\
\hline Human Kinetics and Ergonomics & $\begin{array}{l}\text { Mrs June McDougall } \\
\text { j.mcdougall@ru.ac.za. }\end{array}$ \\
\hline
\end{tabular}




\begin{tabular}{|c|c|}
\hline Ichthyology and Fisheries Science & $\begin{array}{l}\text { Mrs Yvain Erasmus } \\
\text { y.erasmus@ru.ac.za }\end{array}$ \\
\hline Information Systems & $\begin{array}{l}\text { Ms Michelle Coupe } \\
\text { m.coupe@ru.ac.za }\end{array}$ \\
\hline Journalism \& Media Studies & $\begin{array}{l}\text { Miss Marissa van As } \\
\text { m.vanas@ru.ac.za }\end{array}$ \\
\hline Law & Mr Chad Gill c.gill@ru.ac.za \\
\hline Literary Studies in English & $\begin{array}{l}\text { Ms Siphokazi Khanyile } \\
\text { s.khanyile@ru.ac.za }\end{array}$ \\
\hline Management & $\begin{array}{l}\text { Ms Naomi Walton } \\
\text { n.walton@ru.ac.za }\end{array}$ \\
\hline Mathematics (Pure and Applied) & $\begin{array}{l}\text { Mr Loyisa Nkayi } \\
\text { I.nkayi@ru.ac.za }\end{array}$ \\
\hline Modern Fiction & $\begin{array}{l}\text { Mrs Hannelie Campher } \\
\text { h.rielly@ru.ac.za }\end{array}$ \\
\hline Music \& Musicology & $\begin{array}{l}\text { Ms Kanyi Swartbooi } \\
\text { k.swartbooi@ru.ac.za }\end{array}$ \\
\hline Pharmacy & $\begin{array}{l}\text { Mrs Tanya Kent } \\
\text { t.kent@ru.ac.za }\end{array}$ \\
\hline Philosophy & $\begin{array}{l}\text { Mrs Cherron Scheepers } \\
\text { c.duiker@ru.ac.za }\end{array}$ \\
\hline Physics and Electronics & $\begin{array}{l}\text { Ms Ncebakazi Ntsokota } \\
\text { n.ntsokota@ru.ac.za }\end{array}$ \\
\hline Political and International Studies & $\begin{array}{l}\text { Mrs Sibulele Duba } \\
\text { sibulele.duba@ru.ac.za }\end{array}$ \\
\hline
\end{tabular}




\begin{tabular}{|l|l|}
\hline Psychology & $\begin{array}{l}\text { Mrs Vuyo Kofi } \\
\text { v.kofi@ru.ac.za }\end{array}$ \\
\hline School of Languages & $\begin{array}{l}\text { Mrs Hannelie Campher } \\
\text { h.rielly@ru.ac.za }\end{array}$ \\
\hline Sociology & $\begin{array}{l}\text { Mrs Juanita Fuller } \\
\text { j.fuller@ru.ac.za }\end{array}$ \\
\hline Statistics & $\begin{array}{l}\text { Mrs Rene Zimmerman } \\
\text { r.zimmerman@ru.ac.za }\end{array}$ \\
\hline & $\begin{array}{l}\text { Ms Babalwa Booi } \\
\text { b.booi@ru.ac.za }\end{array}$ \\
\hline Zoology and Entomology
\end{tabular}

